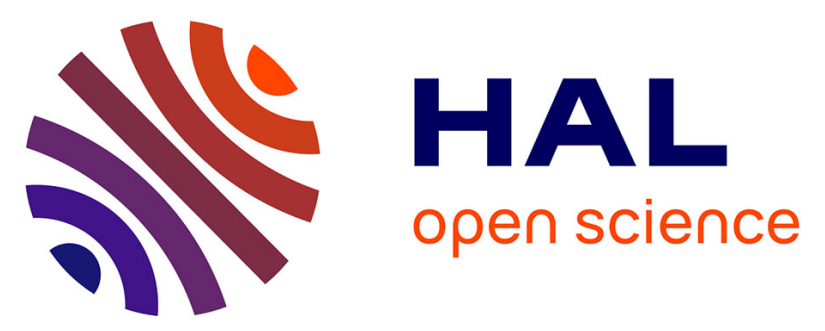

\title{
Calculation of 6D Atomic Surfaces from a Given Approximant Crystalline Structure Using Approximate Icosahedral Periodic Tilings \\ J.-L. Verger-Gaugry
}

\section{- To cite this version:}

J.-L. Verger-Gaugry. Calculation of 6D Atomic Surfaces from a Given Approximant Crystalline Structure Using Approximate Icosahedral Periodic Tilings. Springer Proceedings in Physics, 1990, Number Theory and Physics. Proceedings of the Winter School, Les Houches, France, March 7-16, 1989, 47, pp.128-137. 10.1007/978-3-642-75405-0_12 . hal-03135318

\author{
HAL Id: hal-03135318 \\ https://hal.science/hal-03135318
}

Submitted on 13 Feb 2021

HAL is a multi-disciplinary open access archive for the deposit and dissemination of scientific research documents, whether they are published or not. The documents may come from teaching and research institutions in France or abroad, or from public or private research centers.
L'archive ouverte pluridisciplinaire HAL, est destinée au dépôt et à la diffusion de documents scientifiques de niveau recherche, publiés ou non, émanant des établissements d'enseignement et de recherche français ou étrangers, des laboratoires publics ou privés. 


\title{
Calculation of 6D Atomic Surfaces from a Given Approximant Crystalline Structure Using Approximate Icosahedral Periodic Tilings
}

\author{
J.-L. Verger-Gaugry \\ LTPCM/ENSEEG/INPG (CNRS UA 29), BP 75, Domaine Universitaire, 38402 Saint \\ Martin d'Hères, France
}

\begin{abstract}
Approximant structures of quasicrystals such as $\alpha-\mathrm{A} 1 \mathrm{MnSi}, \mathrm{R}-\mathrm{A} 1 \mathrm{LiCu}$ and $\mathrm{c}\left(\mathrm{Ti}_{2} \mathrm{Ni}\right)$ are well described while atomic organization laws in quasicrystals of similar composition are still unknown. We suggest a method based on the projection method from $\mathbb{R}^{6}$ to calculate, in a general way, the 6D atomic surfaces associated with a given approximant structure, of known atomic decoration, of any Bravais lattice, with respect to the geometrical features inside the unit cell and the distortions in Fourier space. From these atomic surfaces, we deduce three decorated 3D quasilattices of possibly different icosahedral Bravais lattice type $(\mathrm{P}, \mathrm{F}, \mathrm{I})$ and lattice parameters, which in general present aperiodic density and stoichiometry fluctuations.
\end{abstract}




\section{Introduction}

Structure determination of quasicrystals is now one of the main goals in the field of quasicrystals. In order to find the atomic decoration of the icosahedral phase, at first in A1Mn and AlMnSi since Shechtmann et al. /1/ discovered the $i$-phase in the AlMn system in 1984, and subsequently in many other systems, several methods were followed $/ 2 /$.

One experimental method consists in doing a Patterson Fourier analysis in 3 and 6 dimensions of the neutron or X-ray diffraction data on the quasicrystalline phase investigated. Millimetric-sized monoquasicrystalline particles or powders composed only (or essentially) of the quasicrystalline phase are required. Therefore, only a few systems can be tested, such as AlMnSi $/ 3,4 /$, AlLiCu, AlFeCu $/ 5,6 /$, many other systems exhibiting too small a volume fraction of quasicrystals and presenting of ten quasicrystalline particles of too small dimensions, typically micron-sized, in a multi-phased matrix. The resulting Patterson function is represented in 20 sections of $R^{6}$ and shows "continuous" distributions of atoms which reflects the atomic organization in the $i$-phase.

Another experimental method, which necessitates the same requirements as for the feasibility of the quasicrystaline phase in large volume fractions as above, consists in calculating partial pair distribution functions using neutron or $x$-ray diffusion data, as in the case of disordered systems $/ 7,8 /$. By this method, atomic sur faces can sometimes be determined but within a certain accuracy, assuming confidence in the phase reconstruction procedure.

The Patterson approach leads to results which have to be completed by a 60 modelization, that is an explicit allocation of atoms to sets of sites if we consider here that atoms in 6 dimensions are ponctual entities, or to elongated continuous distributions in 30 subspaces in $R^{6}$ in the section framework $/ 9,10 \%$. 0ther experimental methods, such as electron microscopy, provide useful insights into the decoration of the $i$-phase, and a useful basis for modelization as well /11-15/.

Another class of methods, "global" methods, assumes that icosahedral units, such as Mackay's icosahedra, are located at each vertex of a perfect 30 quasilattice $/ 2$, 16/. Icosahedral units are glued in order to form a compact assembly of atoms presenting globally the icosahedral symmetry. Local problems are encountered in the use of such methods: density, resemblance of local environments in approximant structures and $i$-phase, as in AlMnSi,..., and such formal constructions are not yet satisfactory. 
Other methods, "local" methods, start from the principle that an approximant structure has a unit cell which can be partitioned with the same tiles as those encountered in the quasilattice, the non-distorted prolate and oblate rhombohedra, such that atoms are positioned always at equivalent places in each kind of tile, ip respective of the orientation of the tiles in the unit cell. The quasicrystal is then formed by the tiling with both rhombohedra now decorated and placed quasiperiodically $/ 17 /$. This approach has the advantage of keeping the same local clusters in both the crystalline approximant phase and the icosahedral phase. However, some constraints arise : matching rules $/ 18 /$ have to be satisfied, and it is clear that many structures have no reason a priori to be tiled by such non-distorted polyhedra in the way described above. This led some authors to slightly distort local clusters for the matching rules be satisfied $/ 19 /$ to generate quasicrystals.

Because of the existence of a large number of systems in which the $j$-phase forms, under the form of micron-sized particles which cannot be investigated by the methods mentioned above, but only by electron microscopy to obtain reliable diffraction data, there is therefore the need to develop methods to generate quasicrystals based on what we know about approximant structures. This approach is in some sense followed experimentally by Kuo / 20/ since Frank-Kasper and related phases are considered to be approximant phases, and the feasibility of quasicrystals in systems in which such phases form is an attractive challenge $/ 21 /$.

Here, we suggest a method making use of rational cuts in $\mathrm{R}^{6}$ to obtain directly atomic surfaces calculated from the known atomic decoration of a given approximant structure. 60 atoms are considered here as ponctual entities. The present method is new, though the general framework is the projection method $/ 22 \%$, and is not based on a rigid geometry model. The general purpose of this work is to go from approximant structures towards quasicrystals via approximant tiling properties.

\section{Embedding of physical space $E$ in $\mathbb{R}^{6}$}

\subsection{Real and integral (over $\mathbb{Z}$ ) linear representations of the icosahedral group}

Let $G$ the icosahedral group, $|G|=120$. Recalling $/ 22 /$, we take a real linear representation of $G, P: G+G L\left(R^{6}\right), g+P(g)$ such that it is faithful, leaves invariant a symmetric definite positive bilinear form, and that l) it is realizable over $\mathbb{Q}$, which is equivalent to the existence of a G-invariant lattice, 2) $\mathbb{R}^{6}$ is the orthogonal sum of two three-dimensional subspaces endowed with non equivalent actions of $G$, that is non isomorphic simple $R(G)$-modules, $E$ and $E_{4}$. E represents physical space and is the image of the irrational projector $P_{n}$ given by the following matrix in the canonical basis of $\mathbf{R}^{6}$ :

$$
P_{1}=1 / 2 \sqrt{5}\left(\begin{array}{rrrrrr}
\overline{5} & 1 & 1 & 1 & 1 & 1 \\
1 & \sqrt{5} & 1 & -1 & -1 & 1 \\
1 & 1 & \sqrt{5} & 1 & -1 & -1 \\
1 & -1 & 1 & \sqrt{5} & 1 & -1 \\
1 & -1 & -1 & 1 & \sqrt{5} & 1 \\
1 & 1 & -1 & -1 & 1 & \sqrt{5}
\end{array}\right)
$$

Let us call $P_{\perp}=I d-P_{n}, P_{n}: G \rightarrow G L(E)$ the real subrepresentation of $\rho$, and $L$ the generic notation for a G-invariant lattice in $\mathbb{R}^{6}$. Following $/ 23 /$, we note $P$ the primitive lattice $\sqrt{2} \boldsymbol{Z}^{6}$, where the normalization factor is just used to normalize the images of the vectors $e_{i}$, with $\sqrt{2} \mathbb{Z}^{6}=\sum \mathbb{Z} e_{j}$, ie $\left\|P_{n}\left(e_{j}\right)\right\|=1$ for $i=1$ to 6 . $P_{n}$ and $P_{4}$ are orthogonal projectors. For a generic vector $x$ in $\mathbb{R}^{6}$, we call $x_{n}$ or $x^{\prime \prime}$ its projection on $E$ by $P_{n}$ (resp. $x_{1}$ or $x^{1}$ on $E_{1}$ by $P_{1}$ ). When restricted to $\Sigma Q e_{1}$, for $i$ $=1$ to $6, P_{n}$ and $P_{\perp}$ are $Q-i$ somorphisms onto their images, $\Sigma Q_{. j}^{\|}$, resp. $\Sigma$ Qe $e_{j}^{+}$. The 3-faces of the 6 -cubes of $L=P$ are projected onto both rhombohedra known as the prolate and oblate rhombohedra. Whatever $L$ is, the sets $P_{n}(L)$ and $P_{1}(L)$ are dense and uniformly dense in $E$, resp. $E_{1} .\left(e_{i} / \sqrt{2} / i=1\right.$ to 6$\}$ is the canonical basis of $\mathbb{R}^{6}$. 


\subsection{Strip algorithm}

Assume at first $L=P$. In order to tile aperiodically $E$ with only two types of tiles, an open strip E+TR is placed around $E$ in $\mathbb{R}^{6}$ to select nodes of $L$. TR denotes here the image by $P_{n}$ of the open elementary 6 -cube of the lattice $L=P$ at the origin. The quasilattice formed by $L$ and $T R$ is the set of points $\left\{x_{11} / x \in L, x_{\perp} \varepsilon T R\right\}$. This quasilattice can be seen more geometrically by introducing links between points: $n_{m}$ and $m_{m}$ are linked by an edge in the quasilattice iff $n-m$ is one of the 12 vectors te. This provides the edges of rhombohedra and the quasilattice appears as a true tiling with both types of rhombohedra. TR is called "cut volume, or window or pro$f_{i l e "}$ as usual. We call it now TR(P). When $L$ is a G-invariant lattice in $R^{6}$, we call $\operatorname{TR}(L)$ the image by $P_{+}$of an open elementary unit cell associated with the origin. The terminology TR comes from the fact that any elementary 6-cube of L=P projects on a triacontahedron in $E_{\perp}$. With $L$ and $T R(L)$, we can form quasilattices as in the case of $L=P$. More generally, we can take any bounded $G$-invariant convex domain $K$ in $E_{\perp}$ to generate a quasilattice by setting the strip $E+K$ around $E$, selecting nodes in a G-invariant lattice $L$ and projecting them on $E$ by $P_{n}$.

The Fourier transform of a quasilattice has the property that it is G-invariant; its support is dense and, in any bounded domain in reciprocal space, the number of peaks of intensity above a given strictly positive value is finite $/ 23 /$.

The present method is based on the fact that any radiation interacting with matter will only see diffusive center positions and the nature of the diffusive species at these positions but not the rhombohedra edges, which remain, for the incident beam, an abstraction. Therefore, in the following, we shall mainly use vertices and not the underlying geometrical description of the tilings with polyhedra arising from projections of some faces of a G-invariant lattice in $\mathrm{R}^{6}$. We shall adopt a similar attitude for periodic tilings generated by the projection method.

\subsection{G - invariant lattices in $\mathbf{R}^{6}$}

Let us define as usual the lattices $/ 23,24,25 /: P=\sqrt{2 Z^{6}}=\Sigma Z e_{i}$, for $i=1$ to 6 , $I=P U(P+\Delta / 2), 2 F=\left\{\Sigma x_{j} e_{j} \varepsilon P / \Sigma x_{j}\right.$ is even, with $i$ running from 1 to 6$\}, D 6=$ $2 F \cup(R F+\Delta / 2), D^{\prime} 6=2 F U\left(2 F+\Delta F^{\prime} / 2\right)$, with $\Delta=e_{1}+e_{2}+e_{3}+e_{4}+e_{5}+e_{6}$ and $\Delta^{\prime}=$ $\Delta-2 e_{6}$, built on the same basis, the $e_{i}$ 's. They are ${ }^{2} G$-invariant and we Rave the following sequence of inclusions:

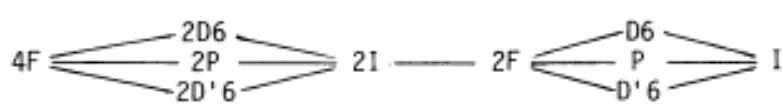

where any G-invariant lattice included in $L_{0}$ and containing $2 L_{0}$ lies in this presentation, where $L_{0}$ is one of the lattices $I, P$ or $2 F$. Since the present method is actually an algorithm which has to be handled by the computer $(\$ 4.2)$, we shall consider only G-invariant lattices included in 1 , and, more precisely, the partially ordered (by inclusion) set $(*)=\{\mathrm{mL} / \mathrm{m} \in \mathrm{N}-\{0\}, \mathrm{L}$ is any of the lattices $\mathrm{P}, \mathrm{I}, 2 \mathrm{~F}$, $\left.D 6, D^{\prime} 6\right\}$. The concept of quasi-homothety classifies these lattices /p 95 in $/ 24 / /$, in particular in $(*)$ : any $G$-invariant lattice $L$ in $R^{6}$, satisfying $2 I \subset L \subset I$, is quasi-homothetic to $I, P$ or $2 F$. Because of such equivalence relations, we shall not consider 06 and $D^{\prime} 6$ in the following. The set $(*)$ will be used to find a G-invariant host lattice for the atomic decoration of a given approximant structure, lifted up in $R^{6}$. The set (*) gives rise to a sequence of quasilattices in $E$ of al ternate icosahedral Bravais lattice, which are of the three types P, I or F, of larger and larger lattice parameters.

The $i$-phase in AIMnSi is of P-type. The first example of F-type icosahedral phase was recently found in the AlFeCu system $/ 26 /$, where diffraction patterns show additional reflections by comparison with those of the $i$-phase in AlMnSi. A possible reordering of atoms in the $i$-phase may occur to explain these diffraction data $/ 6 /$ 
3. Rational cuts and periodic approximant tilings of physical space with pseudoicosahedral symmetry 727 /

\subsection{Preliminary remarks}

As mentioned in 2.2, we start from the need to take more into account vertices in periodic and aperiodic tilings than polyhedra for simple arguments based on the nature of radiation-matter interactions.

We start also with another principle which is of metallurgical nature: in meltspun ribbons, both icosahedral and approximant phases coexist, and particles of both phases of ten stick together. Orientation relationships between them can be determined by electron microscopy by setting a selected area diaphragm on the phase boundary. The existence of the $i$-phase in physical space means that our physical space is embedded in $R^{6}$ in a "rigid way" since it is then stable under the action of $G$. Indeed, in the will to interpret the $i$-phase by a decorated quasilattice, $E$ has to be located in $\mathbb{R}^{6}$ as described in $\S 2$. The existence of approximant phases aiso in $E$ indicate that approximant tilings have to be constructed in $\mathrm{E}$, in view of interpreting approximant structures by approximant tilings. Though in some extent harmless, this remark enables the calculation of the atomic surfaces associated with an approximant structure, whatever its Bravais lattice and space group.

\subsection{Some definitions and basic ideas}

We use the same notations as in $/ 27 /$. A rational cut $E^{\prime}$ is by definition a 30 subspace of $R^{6}$ such that $E^{\prime} \cap I$ is a rank 3 lattice in $E^{\prime}$. We take $I$ instead of $P$ in this definition because it is the densest lattice in the set $(*)$, but any lattice in (*) would be suitable for this definition. We consider 3 -tuples of R-linearly independant vectors $\left\{w_{1}, w_{2}, w_{3}\right\}$ in $E^{\prime} \cap I$. When projected on $E$ by $P_{4}$, they form a basis of $E$; let us call $u_{j}=P_{n}\left(w_{0}\right)$, for $j=1$ to 3 . We denote by $P_{n}$ " the "rational" projector whose image is $E^{\prime}$ and $P_{1}^{\prime}=I d-P_{11}^{\prime}$. Expressed in the $e_{i}^{\prime \prime}$ 's basis, the matrices of $P_{n}^{\prime}$ and $P_{1}$ have coefficients in $Q / 27 \%$. Consequently, the image of any G-invariant lattice of the set $(*)$ by $P_{i}^{\prime}$ on $E^{\prime}$ is discrete and included in $\Sigma Q_{0}=\sqrt{20} 0^{6}$. Because the restriction of $P_{n}$ to $\Sigma Q e_{j}$, with $i=1$ to 6 , is a $Q$-isomorphism onto $\Sigma$ Qe $e_{j}^{\prime}$, with $i=1$ to 6 , the lattice $P_{u}^{j}(L)$ is in one to one correspondence with $i$ ts image $P_{11} P_{11}^{\prime}(L)$ by $P_{n 1}$. By this means, we form two periodic lattices in $E$ : the first one is generated by the $u_{i}$ 's, $i=1$ to 3 , and will fit the crystal system of the approximant structure (cubic, quadratic,...), the other one $P_{n} P_{n}(L)$ will serve as a host lattice for the atomic sites, and is of higher density than the first one. The rational cut $E^{\prime}$ together with the $w_{i}$ "s and the lattice $L$ will not be chosen independently but according to criteria, at first in Fourier space and then in real space, attached to the characteristics of the approximant structure.

Periodic tilings with non distorted rhombohedra. We consider here $L=P$ and $E^{\prime}$ a rational cut. TR denotes the convex set generated by the vectors $P_{i}^{\prime}\left(e_{i}\right)$ in $E_{i}$, slightly translated in $E_{\perp}^{\prime}$ if necessary to be sure of having no point of $P_{\perp}^{\prime}(P)$ on the boundary. As in $/ 23,27 /$, we set the strip $E^{\prime}+T R^{\prime}$ around $E^{\prime}$ to select nodes in $L$ and project them on $E$ by $P_{n}$. This tiling is periodic and constituted by non distorted prolate and oblate rhombohedra. When the volume of TR' increases, the density of selected points in $L$ increases which implies, by projection by $P_{11}$, an enhancement of the density of vertices inside the unit cell (assuming for instance that $\left\{w_{i} / i=1\right.$ to 3$\}$ is a basis of $\left.E^{\prime} \cap P\right\}\left\{u_{1}, u_{2}, u_{3}\right\}$. Rhombohedra are partitioned into smaller and smaller parts. In the present approach, we are looking for coincidence between the true atom positions in the unit cell of the approximant structure and these sets of vertices in order to lift up the decoration on L. But, such a method based on "rational selection and irrational projection" cannot be used with reliability. Indeed, atom positions are determined experimentally within a certain accuracy in the unit cell of the approximant structure and $\mathrm{P}_{\mathrm{n}}(\mathrm{L})$ is dense. Thus, there are infinitely many 
ways to adjust a well-defined cut volume in E: to obtain a covering of these atom positions, and, among them, no canonical way exists. A choice would be arbitrary and would consist in the use of an arbitrary section of the projector $P_{n}$.

Covering by coincidence of an approximant crystalline structure. The idea consists in comparing the crystallographic data of an approximant structure, that is atom positions and their affectation, to both lattices $\Sigma Z_{j}$ and $P_{n}, P_{1}^{\prime}(L)$ where $L$ is $G$-invariant and contains the three vectors $w_{j}$. When $E^{\prime}$ is close to $E$, that is, when, for each $i=1$ to $3,\left\|w_{j}-u_{j}\right\| \ll 1$, the lattice $P_{n} P_{n}^{\prime}(L)$ can be seen as an infinite periodic set of vertices of slightly distorted icosahedral symmetry polyhedra which overlap. As an example, with $L=P$, we have the polyhedron generated by the 12 vectors $\pm P_{n} P_{n}^{\prime}\left(e_{j}\right)$, at the origin, and this situation is repeated at each node in $L$. We can measure in a simple way the proximity of $E^{\prime}$ and $E$ along the direction $u_{i}$ by $a$ rational number $p_{i} / q_{i}$. When the $w_{i}$ 's belong to $P$, say $w_{j}=n_{1 j} e_{1}+\ldots+n_{6 j} e_{6}$, we have: $p_{i} / q_{i}=1 / 2-{ }^{2} N_{2 i} / 4 N_{1 i}$, with $N_{2 i}=$ the sum of the six squares $n_{j}$ and ${ }_{N} N_{1 i}=$ $n_{1 i}\left(-n_{2 i}-n_{3 i}-n_{4 i}-n_{5 i}-n_{6 i}\right)+n_{2 i}\left(-n_{3 i}+n_{4 i}+n_{5 i}-n_{6 i}\right)+n_{3 i}\left(-n_{4 i}+n_{5 i}\right.$ $\left.+n_{6 i}\right)+n_{4 i}\left(-n_{5 i}+n_{6 i}\right)-n_{5 i} n_{6 i}$. For each $i=1$ to 3 , we have the equivalence: $\left\|w_{i}-u_{j}\right\| \ll 1 \Leftrightarrow 0 \ll p_{i} / q_{j}-\tau \ll 1$, where $\tau$ is the golden mean $(1+\sqrt{5}) / 2$.

This comparison between sets of atom positions and discrete periodic sets of vertices is made in the following way: fix once for all $0<\varepsilon \& 1$, for instance $\varepsilon=10^{-2}$ times the smallest lattice parameter $\left\|u_{i}\right\|$ (it is a "variable" constant adapted to the precision we have about atom positions in unit cell), we say that a crystallographic structure is "covered by coincidence by $L$ and $E^{\prime}$ "' when, for each atom position $x$, there exists a unique vertex $y$ belonging to $P_{n} P_{i}^{\prime}(L)$ such that $\|x-y\| \leq \varepsilon$, where $\left\{u_{2}, u_{2}, u_{3}\right\}$ represents the unit cell. In this case, we attribute to $y$ the nature of "the' atom located at the site $x$. For instance, for $c\left(T_{1}{ }^{N i)}\right.$, some vertices will be attributed the "value" Ti, others $\mathrm{Ni}$. We cannot hope that each node $y$ in $P_{n} P_{n}^{\prime}(L)$ is close to an atom position; of course, some $y^{\prime} s$ are deprived of any atom affectation. We give them the value void. In this way, we have built a periodic function on $P_{n} P_{n}^{\prime}(L)$ which takes as many values as there are different types of atoms in the approximant structure, plus one which represents the void affectation. Of course, there is no algebraic structure on the set of values taken by this function.

Because of the algebraic properties of $P_{n}$ and $P_{i}$, this 30 periodic function on $P_{n} P_{n}^{\prime}(L)$ can be uniquely lifted up as a 60 periodic function on $L$. In other words, we can lift up the atomic decoration of the approximant crystalline structure in $R^{6}$, on $L$, once the covering by coincidence of the approximant structure is chosen; that is, we have to decide which $E^{\prime}$ subspaces, which $w_{j}$ 's, and which lattices $L$ have to be used, with respect to the crystallographic data of the approximant structure we consider. This is the purpose of the algorithm suggested in $\$ 4.2$. The atomic decoration of the lattice $L$ is computed as follows: $y$ in P.P. $(L)$ has the same atomic affectation as $z$ in $P_{w}(L)$, where $z$ is the unique point such that $z_{n}=y$. Any $x$ in $L$ such that $P_{i}^{\prime}(x)=z$ receives the same atomic affectation as $y$.

Let us call $\left\{t_{1}, t_{2}, t_{3}\right\}$ a basis for the trace lattice $E_{1} \cap L$. We call $D L$ (for Decoration Lattice) the subiattice of $L$ bearing all the information about the atomic decoration of the approximant structure: it is defined by its generators $\left\{t_{1}, t_{2}\right.$, $\left.t_{3}, w_{1}, w_{2}, w_{3}\right\}$ and its fundamental domain contains all atom positions lifted up in $R 6$ on $L$. Note that the $w_{j}$ 's are taken in $E^{\prime} \cap L$ but do not constitute necessarily a basis of this trace lattice in E. This latice is generally not stable under $G$ and its fundamental domain, together with its collection of atoms, cannot be considered as an atomic surface in $R^{6}(\$ 4.1)$.

\subsection{Pseudo-icosahedral symmetry}

As above, let us consider a G-invariant lattice $L$ and $E$ ' generated by the $w_{3}$ 's. The lattice $\Sigma \Sigma u_{i}$, with $i=1$ to 3 , admits a reciprocal lattice whose generators $u_{j}^{*}$ are defined by $u_{i} \cdot u_{i}^{*}=\delta_{i j}$, in $E$. The $u_{i}^{*}$ 's are therefore $0-1$ inear combinations of the $e_{i n}$ 's and are lifted ${ }^{1} p$ uniquely in $R^{6}$. Let us call $w_{i}^{*}$ the vectors such that $P_{n}\left(w_{i j}^{*}\right)$ $=u_{i}^{*}$ for $i=1$ to $3 . H=\Sigma Z w_{j}^{*}$, with $i=1$ to 3 , is now the reciprocal lattice of the tiling, viewed in $R^{6}$, given by $E^{\prime}$ and the $w_{i}^{\prime}$ 's. 
In $/ 27 /$, we have tiled the unit cell $\left\{u_{i}\right\}$ of the approximant tiling by non distorted rhombohedra by setting a strip (TR' $\left.+E^{\prime}\right)$ around $E^{\prime}$ and we have analyzed the pseudo-icosahedral character of diffraction peaks in Fourier space with a local potential equal to zero everywhere except at the vertices of rhombohedra where we take it equal to 1 . Here, the unit cell is tiled periodically by the lattice $P_{n} P_{i l}^{\prime}(L)$. With the same local potential form, conclusions can be shown to be identical to Prop. 2 in /27/: pseudo-icosahedral symmetry in (3D) reciprocal space can be obtained iff $E^{\prime}$ lies within a narrow 60 solid angle around $E$.

When this condition is satisfied for $E^{\prime}$, intense peaks are aligned on a lattice in reciprocal space and present a pseudo-icosahedral character, that is, positions and intensities of peaks are almost invariant under $G$ (simultaneous double ${ }_{6}$ approximation). Conversely, such an almost invariance allows us to locate $E^{\prime}$ in $\mathbb{R}^{6}$, close to $E$.

Let us recall briefly how pseudo-icosahedral symmetry is described: $G$ acts on the structure factor via the module of the Fourier transform of the set of vertices within the unit cell of the periodic tiling and the phase of the FT is taken into account by homogeneous and nonhomogeneous Diophantine approximation / $28 /$ with 120.5 linear forms describing the action of $G$, and $s$ the number of vertices of the lattice $P_{n} P_{n}(L)$ inside the unit cell. This provides almost invariance in intensities.

Diophantine approximation gives also the existence of a vector $g$ in the G-invariant primitive lattice in $\mathbb{R}^{6}$ generated by the icosahedral orbit of $H^{\prime}$ and its projection on $E_{1}, P_{1}(H)$; the set of exact icosahedral positions of intense peaks (at a given distance of the origin) in reciprocal space is $P_{n}(G) P_{n}(g)$. The corresponding set of true Bragg peak positions is $p_{n}(G) P_{n}\left(g^{H}\right)$, where $g^{H}$ means the projection of $g$ on $\mathrm{H}$ parallel to $\mathrm{E}$.

In this paper, when we speak of an approximant tiling, $E^{\prime}, w_{i}$ 's, we consider that it arises from a rational cut $E^{\prime}$ lying in a narrow solid angle around $E$ of predefinite aperture, for instance $\left\{v \in \mathbb{R}^{6} /\left\|v_{\perp}\right\| /\left\|v_{n}\right\|<5,10^{-2}\right\}$.

When we speak of an approximant structure, it is to indicate that we consider a crystalline phase (for instance a FK phase) and that the experimentally observed distribution of Bragg peaks presents, at a certain distance from the origin in reciprocal space, a pseudo-icosahedral character. If we want to find an approximant tiling $E^{\prime}$, $w_{\text {. ' }} s$, 'which covers by coincidence an approximant structure, $E^{\prime}$ has first to be chosen in a narrow solid angle around $\mathrm{E}$. This is only a consequence of the above proposition. The aperture angle of the solid angle around $\mathrm{E}$ can be computed from the observed maximal distortions of diffraction spots.

\subsection{Distortions and indexation of distortions}

With the same notations, the distortions of Bragg peaks, attached to the exact icosahedral positions given above, are: dis $=\rho_{n}(t) P_{n}\left(g^{H^{\prime}}-g\right)$, with $t$ running in $G$.

Assume that for each Bragg peak, the intensity is slightly spread in a spherical way, as in a Gaussian distribution centered at the peak position. When the approximant tiling is oriented along pseudo $-5,-2,-3$ symmetry elements under the beam, we then observe misalignments and shifts of diffraction peaks in 20 sections in Fourier space, as in electron microscopy in planes tangent to the Ewald sphere. Actually, we observe the projections of the distortion vectors dist on the observation plane.

The experimental situation concerning distortions is far from being simple, with complex shapes and splitting of peaks /29,30/. Knowles/31/ made an analysis of this problem in the AlLiCu system. Some authors interpret peak splitting as a consequence of a macroscopically multitwinned structure built on an approximant tiling which, when endowed with atoms, gives rise to a rhombohedral structure of space group $R 3 \mathrm{~m}$ $/ 32 /$. We limit ourselves to approximant structures with well punctuated Bragg peaks. Distortions can be indexed by 6 integers, which are the coordinates of the vectors dist in the basis (= canonical basis of $\mathbb{R}^{6}$ up to a scaling factor) of the primitive lattice generated by $\rho(G) H$ and $P_{L}(\rho(G) H)$. The symmetry operations of the set of distortions can be computed as well as those which result after projection of the distortions on a 20 "observation plane". 


\subsection{Atomic surfaces}

An atomic surface is here by definition the irreducible fundamental domain of a Ginvariant lattice $L$ in the set $(*)$, punctuated by atoms (the terminology "surface" is of course not correct). This means that it is a finite collection of atoms in $\mathbb{R}^{6}$, on the lattice I, which is attached to one node of $L$ ( $i t$ is usual in three dimensions for a motif), for which there exists no other lattice in (*) containing $L$, and different from L, having a strictly smaller part of this collection as a motif. In other words, we want $L$ to be effectively the icosahedral Bravais lattice of the quasicrystal formed in $E$ from this atomic decoration of $\mathbb{R}^{6}$.

Assume now $L=1, P$ or $2 F$ and that we have found a "good covering by coincidence" of the investigated approximant structure; a dense periodic atomic decoration is now set up on L, and admits as set of translation vectors the lattice we have called DL ( $D L$ depends upon the choice of $L$ ). Calculating the atomic surfaces associated with this approximant structure then means two things:

1) Firstly, the determination of a G-invariant lattice in (*) whose fundamental domain is compatible with the atomic decoration of $\mathbb{R}^{6}$. This signifies that we are looking for the densest and greatest G-invariant lattice in $(*)$ included in DL. The fact that $\mathrm{DL}$ contains G-invariant lattices in (*) comes from Lemma 2, p66 in $/ 33 /$; indeed, if $m$ denotes the covolume of $\mathrm{DL}$ in $\mathrm{L}$, we have $\mathrm{L} \supset \mathrm{DL} \supset \mathrm{mL}$, and since the covolume is an integer, $\mathrm{mL}$ belongs to $\left({ }^{*}\right)$. Let us call GDL the densest and greatest element of $(*)$ included in $\mathrm{DL}$; we have: $\mathrm{I} \supset \mathrm{L} \supset \mathrm{DL} \supset \mathrm{GDL} \supset \mathrm{mL}$. From $\S 2.3$, we know that if there are several possibilities for GDL, they are G-equivalent and therefore give rise to the same decorated quasicrystal in $E$ by projection (by $P_{u}$ ). Of course, GDL can be of P, I or F symmetry type, different from that of L; since this construction makes it the icosahedral Bravais lattice of the quasicrystal we form, its characterization gives directly the symetry type of the quasicrystal built with $L$ and the approximant crystalline structure. The above sequence of inclusions allows the determination of the generators of GDL by the computer by investigating elements of ( $*$ ) between $\mathrm{L}$ and $\mathrm{mL}$ which are included in $\mathrm{DL}$ ( $\mathrm{m}$ is the ratio of the volumes of the unit cells of $\mathrm{DL}$ and $\mathrm{L}$ ).

2) Secondly, the calculation of the atom positions within the unit cell of GDL from those which lie inside a fundamental domain of DL. It amounts to a change of coordinates system for the (6D) atom positions within a unit cell of GDL. Such a decorated unit cell of GDL is an atomic surface in the sense defined above.

The fundamental domain of DL is included in that of the lattice GDL as many times as the value of the covolume (let us call it v; it is an integer) of GDL in DL; this gives a "multiplicity", $=\mathrm{v}$, to each atom and each atomic cluster of the approximant structure lifted up in $\mathbb{R}^{6}$ in the construction process of the quasilattice once $L$ is given. We limit ourselves to $L=P, I$ or $2 F$ because of the classification up to quasi-homothety of the elements of $(*)$. For each L, we obtain a lattice GDL and therefore three quasicrystals, which can be different.

\subsection{Algorithm for the determination of a suitable covering by coincidence of a given} approximant structure

On one hand, we have an approximant crystalline structure, such as a Frank-Kasper phase, which exhibits a pseudo-icosahedral character at a certain distance of the origin in Fourier space, and a large fraction of atom sites with coordination 12, centers of generally slightly distorted icosahedra which are connected by interpenetration or by sharing edges or vertices $/ 20,34 /$. On the other hand, any rational cut. $E^{\prime}$ in a narrow $6 \mathrm{D}$ solid angle around $\mathrm{E}$ gives rise to pseudo-icosahedral periodic tilings in $E$ in which superimposed imbricated slightly distorted icosahedra of various sizes can be found. If $P_{i}^{\prime}$ is the projector associated with $E^{\prime}$, the set of vertices of these icosahedra is the lattice $P_{n} P_{i 1}(L)$, with $L=P$, I or $2 F(\xi 3.2)$.

We would like to adapt an approximant tiling to an approximant structure; that is once $L$ is fixed (step 0 ), we are looking for a rational rank 3 projector $P_{n}^{\prime \prime}$ of ima- 
ge called $E^{\prime}$, and three linearly independent vectors, the $w_{i}$ 's, such that the covolume of the lattice $\Sigma \mathbb{Z} u_{j}, i=1$ to 3 , in $P_{u} P_{n}(L)$ ( = ratio of their respective fundamental domain volumes ${ }^{\prime}$ is as small as possible and that $P_{n} P_{n}(L)$ is maximally occupied by the atoms of the approximant structure (by coincidence), with $w_{j} E E^{\prime} \cap L$. This procedure ensures a maximum occupation of $L$. Such rational cuts $E^{\prime}$ of course exist and we suggest the following algorithm to find them by computer:

step 0 : choice of $L=P, I$ or $2 F$.

step 1: (rough fit of maximal distortions in Fourier space)choice of a narrow solid angle $\delta \Omega$ around $E$ in $\mathbb{R}^{6}$.

step 2 : list the rational cuts $E^{\prime}, w_{j}$ 's, inside $\delta \Omega$ such that $w_{j} \in E^{\prime} \cap L$ and the $u_{j}$ 's with $u_{j}=P_{n}\left(w_{j}\right)$, represent the unit celi up to a scaling factor, of the approximant structure, whatever its Bravais lattice. Practically, we fit the angle relations between the $u_{j}$ 's as they are in the crystal system and both ratios of lattice parameters $\left\|u_{2}\right\| / \eta_{i u_{1} \|,}\left\|u_{3}\right\| /\left\|u_{1}\right\|$ to their analogues b/a, c/a. The projection method imposes that both squares $(b / a)^{2}$ and $(c / a)^{2}$ should belong to $\boldsymbol{Z}[(1+\sqrt{5}) / 2]$ whereas the se elements naturally belong to $Q$. Since $\mathbb{Q}$ and $\mathbb{Z}[(1+\sqrt{5}) / 2]$ are both dense in $\mathbb{R}$, there is no difficulty to express these ratios as elements of $z[(1+\sqrt{5}) / 2]$, by taking approximants. Rational cuts are classified by increasing density of points of $P_{n} P_{u}^{\prime}(L)$ inside the unit cell $\left\{u_{j}\right\}$, with their associated projectors $P_{n}$ and the three rational numbers $\mathrm{p}_{i} / \mathrm{q}_{j}$ reflecting pseudo-icosahedral symmetry.

step 3 : take the first $E^{\prime}$ in the list and compare $P_{n} P_{11}^{\prime}(L)$ with the atom positions in the unit cell of the structure. If a covering by coincidence is not performed, we take the following rational cut $E^{\prime}, w_{i}$ 's, in the list and make again the comparison. More algebraically, coincidence means that the fit takes into account atom orbits under the action of the space group of the structure, and that positional parameters are approximated by elements of $z[(1+\sqrt{5}) / 2]$.

When a covering by coincidence is found, we have modelized the approximant structure; experimental distortions are automatically fitted. We now build the atomic surfaces. In short, to summarize, we have:

step 4 : 1ifting of the atomic decoration on $L$ in $\mathbb{R}^{6}$.

step 5 : determination of the decoration lattice $\mathrm{DL}$.

step 6 : determination of the lattice GDL from DL.

step 7 : calculation of the atomic surface associated with the crystalline structure.

step 8 : projection of this atomic decoration on $E$ by a strip method to form a decorated quasicrystal. We discuss this point below (\$ 4.4$)$.

\subsection{Comments}

We make lists of $w_{j}$ 's on L expressed by their coordinates on the basis of the $e_{j}$ 's, within 82 . We take 3 -tuples of them, construct the list of rational cuts. The determination of projector matrices is performed a posteriori once $E^{\prime}$ is defined by the $w_{i}$ 's.

The research of rational cuts in the list (step 3) stops by itself. Indeed, looking for rational cuts of hioher and higher density of points within the unit cell placed on a lattice of greater and greater "mesh" makes coincidence occur after a finite number of iterations at step 3. If we continue the process, we would obtain the same decorations up to scaling factors.

Once a covering by coincidence is determined, the metrics of $R^{6}$ is fixed: the lengths $\left\|u_{j}\right\|$ are rescaled in $\mathrm{nm}$ (they are the iattice parameters), which allows the determination of the lattice parameters of L and GDL. The resulting quasicrystal buit. by this method inherits the local environments of the approximant structure at the same scale, but are undistorted. Indeed, $P_{n}^{\prime}$ and $P_{n}$ are projectors, i,e. contracting operators of $\mathbb{R}^{6}$, and therefore, keep memory of local environments; when a local cluster of the approximant structure is lifted up on L as a local cluster, which does not of course happen systematically, it remains a local cluster when selected by the strip and projected by $P_{n}$ on $E$, that is of perfect icosahedral symmetry when it is "complete", with almost the same distances between atoms as in the approximant structure but undistorted.

It is obvious that a solution $E^{\prime}, w_{4}$ 's, given by the above algorithm depends upon the choice of $L$, for $L=P, I$ or $2 F$. We then form three types of atomic decoration 
and therefore, three quasicrystals of possibly different icosahedral Bravais lattice and complexity $(\$ 4.1)$.

Once we have modelized the given approximant structure by a ${ }_{6}$ ational cut, and made scaling identifications, we can lift up dislocations in $R^{6}$ and study them explicitely within the ceneral framework developed by Kleman $/ 35 /$, and project them on $E$ together with the atomic decoration.

\subsection{Decorated quasilattices and strip geometry}

Assume now that an atomic decoration of $\mathrm{L}$ (with $\mathrm{L}=\mathrm{P}$, I or 2F) is determined which possesses GDL as G-invariant icosahedral Bravais latice. We try to construct a quasicrystal by the strip + projection method in $E$ (using $P_{n}$ ). There are alternative ways to tackle this problem:

1) The first approach consists in setting a G-invariant cut volume $K$ in $E_{+}$in order to select decorated nodes of $L$ and project them on $E$ as usual. The choice of $K$ requires a careful analysis of the short bonds problem which is inherent to the use of a strip $/ 10 /$. The strip is here a cylinder $E+K$ and its surfaces are parallel to $E$. When the strip is adjusted to respect minimal distances between atoms in the quasicrystal, some (infinitely many) parts of the quasicrystal will be compact and others of smalier density. These density fluctuations reflect the variations of density of the atomic surfaces, and are aperiodic since GDL and $E$ are in incommersurate positions. The whole stability of the quasicrystal can then be investigated by Landau theory $/ 36,37 /$. Comparison can be made in this way between the three types of quasicrystals, at 0 Kelvin, and afterwards at various temperatures to eventually predict phase transitions between them. In the system AlMnSi / 38/, "premonitory rearrangements" upon heating in the $i$-phase were observed, which can in fact be interpreted as phase transitions between quasicrystalline phases associated with the same approximant crystalline phase ( $\alpha-A 1 M n S i)$.

This method provides G-invariant power spectra in Fourier space, that is distortions in Fourier space are undistorted, and local configurations, in real space, existing in the approximant structure become parts of perfect icosahedral units.

Density fluctuations can be accompanied by stoichiometry fluctuations at the scale of the lattice parameter of GDL, though an average value of the stoichiometry is obviously not far from that of the approximant structure. Such chemical variations can explain the fine speckling $(=5 \mathrm{~nm})$ observed on the $i$-phase in many systems /39/ by dark field imaging in electron microscopy.

This approach is now followed in the system $\mathrm{TiNi}$, where $\mathrm{c}\left(\mathrm{Ti}_{2} \mathrm{Ni}\right)$ and $i\left(\mathrm{Ti}_{2} \mathrm{Ni}\right) \mathrm{Co}-$ exist, since we have a good knowledge of the distorted icosahedra within the unit cell of $\mathrm{c}\left(\mathrm{Ti}_{2} \mathrm{Ni}\right) / 40,41 /$.

2) The geometry of the strip can be computed in order to obtain everywhere the same compacity in the quasicrystal, respecting minimal distances as well. Such a construction for the strip is not trivial.

\section{Conclusion}

The present work is an attempt to calculate atomic surfaces in $\mathbb{R}^{6}$ associated with a known approximant crystalline structure using periodic tilings supplied by the projection method from $\mathbb{R}^{6}$. Quasicrystals are formed from such atomic decorations of $\mathbf{R}^{6}$. A numerical application of this method is under way in the system TiNi.

\section{Acknowledgments}

A. Loiseau is thanked for having suggested the problem and for providing very useful experimental support. M. Duneau is acknowledged for very stimulating discussions. 
1. D. Shechtmann, I. Blech, D. Gratias, J.W. Cahn, Phys. Rev. Lett. 53, (1984), 1951.

2. D. Gratias, p 83 in $/ 14 /$.

3. J.W. Cahn, D. Gratias, B. Mozer, Phys. Rev. B (to appear).

4. D. Gratias, J.W. Cahn, B. Mozer, Phys. Rev. B 38, (1988), 1643.

5. T. Ishimasa, Y.Fukano, M. Tsuchimori, Phil. Mag. Lett. 58, (1988), 157.

6. F. Gähler, E3 in $/ 15 /$.

7. C. Janot, J.M. Dubois, J. Pannetier, M. de Boissieu, R. Fruchart, p 107 in /14/.

8. B. Bouchet-Fabre, M. Laridjani, A. Chenoufi, J. Dixmier, p 136 in $/ 14 /$.

9. P. Bak, Scripta Met. 20, (1986), 1199.

10. M. Duneau, C. Oguey, preprint 1988.

11. P.J. Steinhardt, S. Ostlund, The Physics of Quasicrystals (World Scientific), Singapore, (1987).

12. D. Gratias, L. Michel, Int. Workshop on Aperiodic Crystals, J. Physique France, Colloque C3, 47, (1986).

13. K.H. Kuo, Proc. of an Int. Conf. on Quasicrystals, Beijing, Mat. Sci. Forum, 2224, (1987)

14. C. Janot, J.M. Dubois, Proc. of the ILL/Codest Workshop Quasicrystalline Materials, Grenoble, World Scientific, Singapore, (1988).

15. J.M. Dubois, Troisième Colloque sur les quasicristaux, INPL, Vandoeuvre les Nancy, (1989).

16. D. Gratias, p 83 in $/ 21 /$

17. C.L. Henley, V. Elser, Phi1. Mag. B 54, (1986), L101.

18. A. Katz, p 195 in $/ 14 \%$.

19. M. Audier, P. Guyot, p 405 in $/ 12 /$.

20. K.H.Kuo, p 131 in $/ 13 /$.

21. C. Godrèche, Du cristal à l'amorphe, Les Editions de Physique, Les U1is, (1988).

22. M. Duneau, A. Katz, Phys. Rev. Lett. 54, (1985), 2688.

23. M. Duneau, p 157 in $/ 21 /$.

24. D. Martinais, Thèse de Doctorat, Spécialité Mathẻmatiques, Université Paris VII, (June 1988).

25. P. Cartier, C.R. Hebd. Séan. Acad. Sci. B 304, (1987), 789.

26. S. Ebalard, F. Spaepen, J. Mat. Res. (1988), in the press.

27. J.L. Verger-Gaugry, J. Physique France 49, (1988), 1867.

28. R. Descombes, Elements de Thêorie des Nombres, PUF, Paris, (1986).

29. P.A. Bancel, P.A. Heiney, p 341 in $/ 12 /$.

30. K. Urban, J. Mayer, M. Rapp, M. Wilkens, A. Csanady, J. Fidler, p 465 in $/ 12 /$,

31. K.M. Knowles, $p 158$ in $/ 14 \%$.

32. M. Audier, P. Guyot, Acta Met. 36, (1988), 1321.

33. H. Cohn, Advanced Number Theory, Dover, New York, (1980).

34. D.P. Shoemaker, C.B. Shoemaker, p 67 in $/ 13 /$.

35. M. Klèman, $\mathrm{P} 318$ in $/ 14 /$.

36. P.J. Steinhardt, $p 23$ in $/ 13 /$.

37. T. Janssen, p 327 in $/ 14 /$.

38. J.M. Dubois, J.Pannetier, C. Janot, p 311 in $/ 13 /$.

39. K.M. Knowles, W.M. Stobbs, J. Microsc. 146, (1987), 267.

40. A. François, DEA ONERA, Universitê Paris VI, (June 1988)

41. Z. Zhang, H.Q. Ye, K.H. Kuo, Phil. Mag. A 52, (1985), L49. 